\title{
Are Healthcare Professionals Sufficiently Aware of Dental Ethics? Fields Research in Southeast Turkey
}

Elif Pinar Bakir ( $\square$ elifpinarbakir@gmail.com )

Dicle Universitesi https://orcid.org/0000-0003-4011-5091

\section{Seyhmus Bakir}

Dicle university Faculty of Dentistry

\section{Ezgi Sonkaya}

Dicle University Faculty of Dentistry

\section{Samican Ünal Ünal}

Dicle University Faculty Of Dentistry

\section{Research article}

Keywords: Patient rights, legal responsibilities, healthcare personel awareness, medical ethics

Posted Date: September 21st, 2020

DOI: https://doi.org/10.21203/rs.3.rs-50692/v1

License: (c) (i) This work is licensed under a Creative Commons Attribution 4.0 International License. Read Full License

Version of Record: A version of this preprint was published at Biotechnology \& Biotechnological Equipment on January 1st, 2021. See the published version at https://doi.org/10.1080/13102818.2021.1875877. 


\section{Abstract}

Background: The aim of this study was to evaluate the awareness of healthcare personnel in the Dentistry Faculty of Dicle University about "the legal responsibilities of dental practitioners" and "patient rights".

Methods: The study included 150 volunteers who were healthcare workers in the Faculty of Dentistry , Dicle University between June and July 2019. The subjects were randomly selected from five different Professional groups and data were collecte dusing a single data collection form. All items on the form were grade dusing a 5-point Likert -type response. In the statistical analyses, Pearson's Chi-square, Yates Chi-square and the Fisher Exact tests were used.

Results:The questionnaire responses showed that $61.3 \%$ of the study participants had received training on patient rights and $56.7 \%$ had information about the legal responsibilities of dentists. The rate of dentists who had received training was below average and $38.9 \%$ of the dentists reported not having any information about their own legal responsibilities. A positive response was given to the items on the questionnaire by $58 \%$ and a negative responseby $33 \%$. The responses to some items were seen to have been affected by variables such as age, gender and professional group.

Conclusion: Although some confusion was experienced by the healthcare personel about patient rights and the legal responsibilities of dentists, the levels of awareness were evaluated as satisfactory.

\section{Background}

The concept of patient rights can be defined as fulfilling the basic needs and expectations of the patient. These rights are based on showing respect, receiving the best service, being informed correctly, consent for interventional proceedures and patient confidentiality [1]. The first documentation related to patient rights was the Lisbon Declaration published by the World Medical Association in 1981. This declaration includes headings such as confidentiality of information, and the rights of the patient to select a physician, and accept or reject treatment. In Turkey, the"Patient Rights Guidelines" published by the Ministry of Health came into operation in 1998 [2, 3,26].

The basic rules related to patient rights -according to medical ethics- can be summarised as:

-Patients should benefit equally and in the best way from healthcare services. Patients should not be differentiated in respect of gender, race, language, beliefs and economic or social status.

- No individual or institution has the right to remove the patient's right to life. With the exception of medical and legal necessities, the integrity of the body cannot be touched without the consent of the individual. Obtaining informed consent is a prerequisite.

- When it is necessary to explain the risks of diagnosis and there commended treatment services, the patient must be given an example of the medical records.

- The patient must be able to obtain information about the attending physician and other healthcare personnel. The patient has the right to select a physician, and accept or reject or halt treatment. When necessary, the patient must be transferred to another healthcare facility.

- Information related to the patient must be kept confidential. The patient must be able to access communication and religious services. When necessary, the patient must be able to submit a complaint [4].

An increased work load and some economic, social and technological problems can cause problems in healthcare personnel-patient communication. It is a primary duty and responsibility of healthcare personel to pay attention to 
patient rights and to prevent neglect of these rights $[5,6]$. Patients should have sufficient information and sensitivity about their own rights $[7,8]$.

The most important criteria of high-quality healthcare service is sensitivity shown to meeting the needs and expectations of patients and patient satisfaction $[9,10]$. Patient satisfaction is the difference between the service imagined and perceived by the patient [11]. Factors affecting patient satisfaction include the age, gender, education level and income level of the patient, and the educational level of the health care personnel $[12,13]$.

In Turkey, a system protecting patient rights and preventing medical mistakes has still not been fully implemented. Especially during dental services, the predominant view is that patient rights are neglected. However, as a result of incorrect interventional procedures, there are criminal, compensation and administrative responsibilities. Criminal responsibility arises from carelessness and lack of precautions taken by the physician. Compensation responsibility corresponds to financial and emotional damage experienced by the patient and their family. Administrative responsibility is determined with a disciplinary investigation to be conducted by the dental association or institution where the dentist is employed [14].

For dentists to be able to be free of legal liability:

1. The diagnosis and treatment must be made only by the dentist,

2. Treatment applications must be based on medical, social or psychological indications,

3. Written informed consent must be obtained from the patient before interventional procedures,

4. Treatment procedures must be applied with care as required by the dental specialist area, $[4,14]$.

To improve the quality of the services provided, patient satisfaction should be periodically evaluated $[15,16]$. Questionnaire studies applied will contribute to healthcare planning [17]. In recent years, there have been several scientific studies and activities on the subject of patient rights in Turkey. A signficant proportion of these have measured the awareness of patients and physicians $[3,15,18]$. As patients have more communication with healthcare personel other than physicians, and a significant amount of medical and administrative procedures are conducted by these personnel, it is necessary to evaluate their awareness [19].

In accordance with this understanding, the aim of this study was to evaluate the awareness of healthcare personel working in the Dental Faculty of Dicle University about patient rights and the legal responsibilities of dental practitioners.

\section{Methods}

The study was carried out with a total of 150 volunteers(dentist, nurse, Healthcare clerical worker, health technician, and other assistant health personnel)

determined by using random sampling method from five different groups employed at Dicle University Faculty of Dentistry in 2019 between June and July. The survey technique, which is one of the quantitative data collection techniques, was used in our research to measure the level of knowledge of various levels of health professionals about patient rights and legal responsibilities of dentists. In the literature research, the studies related to the subject, which were made to create the scale, were examined by four academicians who are competent in their field in terms of determining the questionnaire questions and using the scale. All staff working in the hospital participated in the questionnaire and care was taken to be willing to participate in the research. Participants were informed about the purpose of the study and their consent was obtained by paying attention to the principle of volunteering. It was also ensured that the information of the participants would be kept confidential. In the research, 150 health personnel were surveyed. 
The responses given by individuals were evaluated according to the regulations of the TDB (Turkish Dental Association), which was adopted by the 14th Ordinary General Assembly of TDB (Turkish Dental Association) and the patient rights guide published by the Ministry of Health, which was launched in 1998, and their awareness was interpreted.

In the research, a data collection form compatible with the Ministry of Health Patient Rights Guidelines was used. Approval for the study was given by the Ethics Committee of Dicle University Faculty of Dentistry (decision no: 2019/3). The questionnaire consists of 25 items in 2 sections. The answers were in the form of positive and negative statements as 5-point Likert-typeresponse: 1 = Strongly agree, 2 = Agree, 3 = Undecided, 4 = Disagree, 5 = Strongly disagree [Table 2]Data obtained in the study were analysed statistically using R version 3.2.3 (2015-12-10), Copyright (C) 2015 The R Foundation for Statistical Computing free software. In the statistical evaluation of categorical variables (number, percentage), the Pearson Chi-square, Yates Chi-square and Fisher Exact tests were used. A confidence interval of $95 \%$ was applied. A value of $p<0.05$ was accepted as statistically signficant. Table 1 . The opinions of the healthcare personnel

Table 1

Descriptive information for health professionals

\begin{tabular}{|c|c|c|c|c|c|c|c|}
\hline & & Dentist & Nurse & $\begin{array}{l}\text { Healthcare } \\
\text { clerical } \\
\text { worker }\end{array}$ & $\begin{array}{l}\text { Healthcare } \\
\text { technician }\end{array}$ & Other & Total \\
\hline \multirow[t]{2}{*}{ Gender } & Women & $\begin{array}{l}52 \\
(\% 34.7)\end{array}$ & $\begin{array}{l}8 \\
(\% 5.4)\end{array}$ & $3(\% 2.0)$ & $8(\% 5.4)$ & $\begin{array}{l}4 \\
(\% 2.7)\end{array}$ & $\begin{array}{l}75 \\
(\% 50.0)\end{array}$ \\
\hline & Men & $\begin{array}{l}61 \\
(\% 40.6)\end{array}$ & $\begin{array}{l}2 \\
(\% 1.3)\end{array}$ & $6(\% 4.0)$ & $5(\% 3.3)$ & $\begin{array}{l}1 \\
(\% 0.6)\end{array}$ & $\begin{array}{l}75 \\
(\% 50.0)\end{array}$ \\
\hline \multirow[t]{4}{*}{ Age } & $18-25$ & $\begin{array}{l}20 \\
(\% 13.3)\end{array}$ & $\begin{array}{l}1 \\
(\% 0.6)\end{array}$ & $1(\% 0.6)$ & $2(\% 1.3)$ & $\begin{array}{l}1 \\
(\% 0.6)\end{array}$ & $\begin{array}{l}25 \\
(\% 16.6)\end{array}$ \\
\hline & $26-35$ & $\begin{array}{l}40 \\
(\% 26.6)\end{array}$ & $\begin{array}{l}2 \\
(\% 1.3)\end{array}$ & $1(\% 0.6)$ & $2(\% 1.3)$ & $1 \% 0.6)$ & $\begin{array}{l}46 \\
(\% 30.7)\end{array}$ \\
\hline & $36-45$ & $\begin{array}{l}25 \\
(\% 16,6)\end{array}$ & $\begin{array}{l}2 \\
(\% 1.3)\end{array}$ & $2(\% 1.3)$ & $4(\% 2.7)$ & $\begin{array}{l}1 \\
(\% 0.6)\end{array}$ & $\begin{array}{l}34 \\
(\% 22.7)\end{array}$ \\
\hline & $\begin{array}{l}46 \text { and } \\
\text { older }\end{array}$ & $\begin{array}{l}28 \\
(\% 18.6)\end{array}$ & $\begin{array}{l}5 \\
(\% 3.3)\end{array}$ & $5(\% 3.3)$ & $5(\% 3.3)$ & $\begin{array}{l}2 \\
(\% 1.3)\end{array}$ & $\begin{array}{l}45 \\
(\% 30.0)\end{array}$ \\
\hline \multirow[t]{2}{*}{$\begin{array}{l}\text { Have you received training } \\
\text { about patient rights? }\end{array}$} & Yes & $\begin{array}{l}62 \\
(\% 41.3)\end{array}$ & $\begin{array}{l}7 \\
(\% 4.6)\end{array}$ & $5(\% 3.3)$ & $7(\% 4.6)$ & $\begin{array}{l}1 \\
(\% 0.6)\end{array}$ & $\begin{array}{l}82 \\
(\% 54.6)\end{array}$ \\
\hline & No & $\begin{array}{l}51 \\
(\% 34.0)\end{array}$ & $\begin{array}{l}3 \\
(\% 2.0)\end{array}$ & $4(\% 2.7)$ & $6(\% 4.0)$ & $\begin{array}{l}4 \\
(\% 2.7)\end{array}$ & $\begin{array}{l}68 \\
(\% 45.4)\end{array}$ \\
\hline \multirow[t]{2}{*}{$\begin{array}{l}\text { Do you know about the legal } \\
\text { responsibilities of dentists }\end{array}$} & Yes & $\begin{array}{l}69 \\
(\% 46.0)\end{array}$ & $\begin{array}{l}4 \\
(\% 2.7)\end{array}$ & $4(\% 2.7)$ & $3(\% 2.0)$ & $\begin{array}{l}1 \\
(\% 0.6)\end{array}$ & $\begin{array}{l}81 \\
(\% 54.0)\end{array}$ \\
\hline & No & $\begin{array}{l}44 \\
(\% 29.3)\end{array}$ & $\begin{array}{l}6 \\
(\% 4.0)\end{array}$ & $5(\% 3.3)$ & $10(\% 6.7)$ & $\begin{array}{l}4 \\
(\% 2.7)\end{array}$ & $\begin{array}{l}69 \\
(\% 46.0)\end{array}$ \\
\hline
\end{tabular}


Table 2

The opinions of the healthcare personnel

\begin{tabular}{|c|c|c|c|c|c|c|c|c|c|c|c|}
\hline \multirow{3}{*}{$\begin{array}{l}\text { Item } \\
\text { No }\end{array}$} & \multirow[t]{3}{*}{ ITEM } & \multicolumn{10}{|c|}{ Responses } \\
\hline & & \multicolumn{2}{|c|}{$\begin{array}{l}\text { I definitely } \\
\text { agree }\end{array}$} & \multicolumn{2}{|c|}{ I agree } & \multicolumn{2}{|c|}{$\begin{array}{l}\text { I am un- } \\
\text { decided }\end{array}$} & \multicolumn{2}{|c|}{$\begin{array}{l}\text { I do not } \\
\text { agree }\end{array}$} & \multicolumn{2}{|c|}{$\begin{array}{l}\text { I defini- } \\
\text { tely do } \\
\text { not agree }\end{array}$} \\
\hline & & $n$ & $\%$ & $\mathrm{n}$ & $\%$ & $\mathrm{n}$ & $\%$ & $\mathrm{n}$ & $\%$ & $n$ & $\%$ \\
\hline 6 & $\begin{array}{l}\text { Every dentist has the authority to } \\
\text { make age determinations }\end{array}$ & 47 & 31.3 & 54 & 36 & 30 & 20 & 15 & 10 & 4 & 2.7 \\
\hline 7 & $\begin{array}{l}\text { The dentist must not takeover a } \\
\text { colleague's patients }\end{array}$ & 93 & 62 & 36 & 24 & 6 & 4 & 9 & 6 & 6 & 4 \\
\hline 8 & $\begin{array}{l}\text { The dentist should give information } \\
\text { about complications to a patient who } \\
\text { is receiving treatment for the first time }\end{array}$ & 118 & 78.6 & 29 & 19.3 & 1 & 0.7 & 1 & 0.7 & 1 & 0.7 \\
\hline 9 & $\begin{array}{l}\text { The dentist can refuse to see a patient } \\
\text { for Professional or personal reasons }\end{array}$ & 67 & 44.6 & 46 & 30.7 & 21 & 14 & 13 & 8.7 & 3 & 2 \\
\hline 10 & $\begin{array}{l}\text { The dentist can leave a patient } \\
\text { without finishing the treatment for } \\
\text { Professional or personal reasons }\end{array}$ & 22 & 14.7 & 24 & 16 & 22 & 14.7 & 57 & 38 & 25 & 16.6 \\
\hline 11 & $\begin{array}{l}\text { The dentist has the authority to print a } \\
\text { notice of thanks in the form of an } \\
\text { advertisement in newspapers }\end{array}$ & 8 & 5.3 & 22 & 14.7 & 67 & 44.7 & 28 & 18.7 & 25 & 16.6 \\
\hline 12 & $\begin{array}{l}\text { The dentist can open a vascular route } \\
\text { for intervention to the patient in } \\
\text { emergency situations }\end{array}$ & 39 & 26 & 50 & 33.3 & 30 & 20 & 18 & 12 & 13 & 8.7 \\
\hline 13 & $\begin{array}{l}\text { The dentist can prescribe drugs for the } \\
\text { patients with a gren prescription }\end{array}$ & 14 & 9.3 & 30 & 20 & 43 & 28.7 & 28 & 18.7 & 35 & 23.3 \\
\hline 14 & $\begin{array}{l}\text { Everydentist can prepare an expert } \\
\text { report in forensic cases. }\end{array}$ & 35 & 23.3 & 42 & 28 & 28 & 18.7 & 31 & 20.7 & 14 & 9.3 \\
\hline 15 & $\begin{array}{l}\text { The dentist can perform treatments } \\
\text { such as botox and dermal fillers } \\
\text { without a specialist certificate }\end{array}$ & 25 & 16.6 & 23 & 15.3 & 19 & 12.7 & 37 & 24.7 & 46 & 30.7 \\
\hline 16 & $\begin{array}{l}\text { The dentist can apply treatments } \\
\text { under general anaesthesia without a } \\
\text { specialist certificate }\end{array}$ & 13 & 8.7 & 19 & 12.7 & 20 & 13.3 & 41 & 27.3 & 57 & 38 \\
\hline 17 & $\begin{array}{l}\text { The dentist can write a sick note for a } \\
\text { patient without a specialist certificate }\end{array}$ & 58 & 38.6 & 49 & 32.7 & 9 & 6 & 18 & 12 & 16 & 10.7 \\
\hline 18 & $\begin{array}{l}\text { The dentist can diagnose and } \\
\text { prescribe drugs for a disease other } \\
\text { than in his own field (diabetes, } \\
\text { hypertension) }\end{array}$ & 8 & 5.3 & 5 & 3.3 & 15 & 10 & 49 & 32.7 & 73 & 48.7 \\
\hline 19 & $\begin{array}{l}\text { Patient consent must be obtained for } \\
\text { every kind of medical intervention, and } \\
\text { the patient must benefit from these }\end{array}$ & 115 & 76.7 & 24 & 16 & 5 & 3.3 & 5 & 3.3 & 1 & 0.7 \\
\hline 20 & $\begin{array}{l}\text { A diagnosis can be with held from a } \\
\text { patient if it is going to worsen a } \\
\text { psychological state orexacerbate the } \\
\text { disease. }\end{array}$ & 24 & 16 & 35 & 23.3 & 32 & 21.4 & 29 & 19.3 & 30 & 20 \\
\hline
\end{tabular}




\begin{tabular}{|c|c|c|c|c|c|c|c|c|c|c|c|}
\hline \multirow{3}{*}{$\begin{array}{l}\text { Item } \\
\text { No }\end{array}$} & \multirow[t]{3}{*}{ ITEM } & \multicolumn{10}{|c|}{ Responses } \\
\hline & & \multicolumn{2}{|c|}{$\begin{array}{l}\text { I definitely } \\
\text { agree }\end{array}$} & \multicolumn{2}{|c|}{ I agree } & \multicolumn{2}{|c|}{$\begin{array}{l}\text { I am un- } \\
\text { decided }\end{array}$} & \multicolumn{2}{|c|}{$\begin{array}{l}\text { I do not } \\
\text { agree }\end{array}$} & \multicolumn{2}{|c|}{$\begin{array}{l}\text { I defini- } \\
\text { tely do } \\
\text { not agree }\end{array}$} \\
\hline & & $\mathrm{n}$ & $\%$ & $n$ & $\%$ & $\mathrm{n}$ & $\%$ & $\mathrm{n}$ & $\%$ & $\mathrm{n}$ & $\%$ \\
\hline 21 & $\begin{array}{l}\text { The patient has the right to know the } \\
\text { identity, position and title of the } \\
\text { dentist and other personel who will } \\
\text { provide the healthcare and has the } \\
\text { right to select and change the personel } \\
\text { who will provide the healthcare }\end{array}$ & 64 & 42.7 & 46 & 30.7 & 18 & 12 & 17 & 11.3 & 5 & 3.3 \\
\hline 22 & $\begin{array}{l}\text { There is a responsibility to give full } \\
\text { and complete information related to } \\
\text { the patient's health }\end{array}$ & 101 & 67.3 & 38 & 25.3 & 3 & 2 & 4 & 2.7 & 4 & 2.7 \\
\hline 23 & $\begin{array}{l}\text { The patient is liable for any damage to } \\
\text { hospital materials }\end{array}$ & 69 & 46 & 25 & 16.6 & 28 & 18.7 & 13 & 8.7 & 15 & 10 \\
\hline 24 & $\begin{array}{l}\text { Medical interventions can only be } \\
\text { made without patient consent } \\
\text { because of medical necessity and } \\
\text { when precautions must be taken in } \\
\text { respect of public health }\end{array}$ & 35 & 23.3 & 45 & 30 & 36 & 24 & 12 & 8 & 22 & 14.7 \\
\hline 25 & $\begin{array}{l}\text { The patient can request all kinds of } \\
\text { information verbally and in writing } \\
\text { related to their health status }\end{array}$ & 89 & 59.3 & 46 & 30.7 & 7 & 4.7 & 5 & 3.3 & 3 & 2 \\
\hline
\end{tabular}


Table 2

Opinions of the healthcare personel according to Professional group

\begin{tabular}{|c|c|c|c|c|c|c|}
\hline \multirow[t]{2}{*}{ Item9 } & \multicolumn{5}{|c|}{ The dentist can refuse to see a patient for Professional or personal reasons. } & \multirow{2}{*}{$\begin{array}{l}\text { Total no of } \\
\text { respondents }\end{array}$} \\
\hline & $\begin{array}{l}\text { I } \\
\text { definitely } \\
\text { agree }\end{array}$ & I agree & I am undecided & $\begin{array}{l}\text { I do } \\
\text { not } \\
\text { agree }\end{array}$ & $\begin{array}{l}\text { I definitely do } \\
\text { not agree }\end{array}$ & \\
\hline Dentist & $54.9 \%$ & $31.9 \%$ & $8 \%$ & $3.5 \%$ & $1.7 \%$ & 113 \\
\hline Nurse & $10 \%$ & $40 \%$ & $30 \%$ & $20 \%$ & $0 \%$ & 10 \\
\hline $\begin{array}{l}\text { Healthcare } \\
\text { clericalworker }\end{array}$ & $11.1 \%$ & $11.1 \%$ & $55.6 \%$ & $11.1 \%$ & $11.1 \%$ & 9 \\
\hline $\begin{array}{l}\text { Healthcare } \\
\text { technician }\end{array}$ & $15.4 \%$ & $38.5 \%$ & $15.4 \%$ & $30.7 \%$ & $0 \%$ & 13 \\
\hline Other & $20 \%$ & $0 \%$ & $40 \%$ & $40 \%$ & $0 \%$ & 5 \\
\hline Total & $44.7 \%$ & $30.7 \%$ & $14 \%$ & $8.7 \%$ & $2 \%$ & 150 \\
\hline \multirow[t]{2}{*}{ Item 19} & \multicolumn{5}{|c|}{$\begin{array}{l}\text { Patient consent must be obtained for every kind of medical intervention and } \\
\text { the patient must benefit from these. }\end{array}$} & \multirow[t]{2}{*}{$\begin{array}{l}\text { Total no of } \\
\text { respondents }\end{array}$} \\
\hline & $\begin{array}{l}\text { Iefinitely } \\
\text { agree }\end{array}$ & I agree & I am undecided & $\begin{array}{l}\text { I do } \\
\text { not } \\
\text { agree }\end{array}$ & $\begin{array}{l}\text { I definitely do } \\
\text { not agree }\end{array}$ & \\
\hline Dentist & $73.5 \%$ & $18.6 \%$ & $3.5 \%$ & $3.5 \%$ & $0.9 \%$ & 113 \\
\hline Nurse & $80 \%$ & $0 \%$ & $10 \%$ & $10 \%$ & $0 \%$ & 10 \\
\hline $\begin{array}{l}\text { Healthcare } \\
\text { clericalworker }\end{array}$ & $77.8 \%$ & $22.2 \%$ & $0 \%$ & $0 \%$ & $0 \%$ & 9 \\
\hline $\begin{array}{l}\text { Healthcare } \\
\text { technician }\end{array}$ & $92.3 \%$ & $7.7 \%$ & $0 \%$ & $0 \%$ & $0 \%$ & 13 \\
\hline Other & $100 \%$ & $0 \%$ & $0 \%$ & $0 \%$ & $0 \%$ & 5 \\
\hline Total & $76.7 \%$ & $16 \%$ & $3.3 \%$ & $3.3 \%$ & $0.7 \%$ & 150 \\
\hline \multirow[t]{2}{*}{ Item 21} & \multicolumn{5}{|c|}{$\begin{array}{l}\text { The patient has the right to know the identity position and title of the dentist } \\
\text { and other personel who will provide the healthcare and has the right to select } \\
\text { and change the personel who will provide the healthcare. }\end{array}$} & \multirow[t]{2}{*}{$\begin{array}{l}\text { Total no of } \\
\text { respondents }\end{array}$} \\
\hline & $\begin{array}{l}\text { definitely } \\
\text { agree }\end{array}$ & I agree & I am undecided & $\begin{array}{l}\text { I do } \\
\text { not } \\
\text { agree }\end{array}$ & $\begin{array}{l}\text { I definitely do } \\
\text { not agree }\end{array}$ & \\
\hline Dentist & $39.8 \%$ & $35.4 \%$ & $10.6 \%$ & $9.7 \%$ & $4.5 \%$ & 113 \\
\hline Nurse & $60 \%$ & $10 \%$ & $10 \%$ & $20 \%$ & $0 \%$ & 10 \\
\hline $\begin{array}{l}\text { Healthcare } \\
\text { clerical } \\
\text { worker }\end{array}$ & $44.5 \%$ & $22.2 \%$ & $0 \%$ & $33.3 \%$ & $0 \%$ & 9 \\
\hline $\begin{array}{l}\text { Healthcare } \\
\text { technician }\end{array}$ & $46.2 \%$ & $15.4 \%$ & $30.8 \%$ & $7.6 \%$ & $0 \%$ & 13 \\
\hline Other & $60 \%$ & $20 \%$ & $20 \%$ & $0 \%$ & $0 \%$ & 5 \\
\hline Total & $42.7 \%$ & $30.7 \%$ & $12 \%$ & $11.3 \%$ & $3.3 \%$ & 150 \\
\hline
\end{tabular}




\begin{tabular}{|c|c|c|c|c|c|c|}
\hline \multirow[t]{2}{*}{ Item 9} & \multicolumn{5}{|c|}{ The dentist can refuse to see a patient for Professional or personal reasons. } & \multirow{2}{*}{$\begin{array}{l}\text { Total no of } \\
\text { respondents }\end{array}$} \\
\hline & $\begin{array}{l}\text { I definitely } \\
\text { agree }\end{array}$ & & decided & $\begin{array}{l}\text { I do } \\
\text { not } \\
\text { agree }\end{array}$ & $\begin{array}{l}\text { I definitely do } \\
\text { not agree }\end{array}$ & \\
\hline \multirow[t]{2}{*}{ Item 24} & \multicolumn{5}{|c|}{$\begin{array}{l}\text { Medical interventions can only be made without patient consent because of } \\
\text { medical necessity and when precautions must be taken in respect of public } \\
\text { health }\end{array}$} & \multirow[t]{2}{*}{$\begin{array}{l}\text { Total no of } \\
\text { respondents }\end{array}$} \\
\hline & I definitely agree & I agree & $\begin{array}{l}\text { I am } \\
\text { undecided }\end{array}$ & $\begin{array}{l}\text { I do not } \\
\text { agree }\end{array}$ & $\begin{array}{l}\text { I definitely do } \\
\text { not agree }\end{array}$ & \\
\hline Dentist & $23.9 \%$ & $33.6 \%$ & $23.9 \%$ & $8.8 \%$ & $9.8 \%$ & 113 \\
\hline Nurse & $20 \%$ & $30 \%$ & $20 \%$ & $10 \%$ & $20 \%$ & 10 \\
\hline $\begin{array}{l}\text { Healthcare } \\
\text { clerical worker }\end{array}$ & $33.3 \%$ & $33.3 \%$ & $33.3 \%$ & $0 \%$ & $0 \%$ & 9 \\
\hline $\begin{array}{l}\text { Healthcare } \\
\text { technician }\end{array}$ & $15.4 \%$ & $7.7 \%$ & $23.1 \%$ & $7.8 \%$ & $46.2 \%$ & 13 \\
\hline Other & $20 \%$ & $0 \%$ & $20 \%$ & $0 \%$ & $60 \%$ & 5 \\
\hline Total & $23.3 \%$ & $30 \%$ & $24 \%$ & $8 \%$ & $14.7 \%$ & 150 \\
\hline
\end{tabular}

\section{Results}

At the end of the study, it was determined that the Professional distribution was not balanced, as dentists comprised $75 \%$ of the study sample [Table 2], males and females were represented equally [Table 3], and more than half the participants were in the 18-25 years age group [Table 4]. 
Table 3

Opinions of the healthcare workers according to gender

\begin{tabular}{|c|c|c|c|c|c|c|}
\hline \multirow[t]{2}{*}{ Item 9} & \multicolumn{5}{|c|}{ The dentist can refuse to see a patient for Professional or personal reasons } & \multirow{2}{*}{$\begin{array}{l}\text { Total no of } \\
\text { respondents }\end{array}$} \\
\hline & $\begin{array}{l}\text { I definitely } \\
\text { agree }\end{array}$ & $\begin{array}{l}\text { I } \\
\text { agree }\end{array}$ & $\begin{array}{l}\text { I am } \\
\text { undecided }\end{array}$ & $\begin{array}{l}\text { I do not } \\
\text { agree }\end{array}$ & $\begin{array}{l}\text { I definitely do not } \\
\text { agree }\end{array}$ & \\
\hline Female & $44 \%$ & $26.7 \%$ & $18.7 \%$ & $10.6 \%$ & $0 \%$ & 75 \\
\hline Male & $45.3 \%$ & $34.7 \%$ & $9.3 \%$ & $6.7 \%$ & $4 \%$ & 75 \\
\hline Total & $44.7 \%$ & $30.7 \%$ & $14 \%$ & $8.7 \%$ & $2 \%$ & 150 \\
\hline \multirow[t]{2}{*}{$\begin{array}{l}\text { Item } \\
15\end{array}$} & \multicolumn{5}{|c|}{$\begin{array}{l}\text { The dentist can perform treatments such as botox and dermal fillers without a } \\
\text { specialist certificate }\end{array}$} & \multirow[t]{2}{*}{$\begin{array}{l}\text { Total no of } \\
\text { respondents }\end{array}$} \\
\hline & $\begin{array}{l}\text { I definitely } \\
\text { agree }\end{array}$ & lagree & $\begin{array}{l}\text { I am } \\
\text { undecided }\end{array}$ & $\begin{array}{l}\text { I do not } \\
\text { agree }\end{array}$ & $\begin{array}{l}\text { I definitely do not } \\
\text { agree }\end{array}$ & \\
\hline Female & $12 \%$ & $12 \%$ & $13.3 \%$ & $28 \%$ & $34.7 \%$ & 75 \\
\hline Male & $21.3 \%$ & $18.7 \%$ & $12 \%$ & $21.3 \%$ & $26.7 \%$ & 75 \\
\hline Total & $16.7 \%$ & $15.3 \%$ & $12.7 \%$ & $24.7 \%$ & $30.7 \%$ & 150 \\
\hline \multirow[t]{2}{*}{$\begin{array}{l}\text { Item } \\
22\end{array}$} & \multicolumn{5}{|c|}{$\begin{array}{l}\text { There is a responsibility to give full and complete information related to the } \\
\text { patient's health. }\end{array}$} & \multirow[t]{2}{*}{$\begin{array}{l}\text { Total no of } \\
\text { respondents }\end{array}$} \\
\hline & $\begin{array}{l}\text { I definitely } \\
\text { agree }\end{array}$ & I & $\begin{array}{l}\text { I am } \\
\text { undecided }\end{array}$ & $\begin{array}{l}\text { I do not } \\
\text { agree }\end{array}$ & $\begin{array}{l}\text { I definitely do not } \\
\text { agree }\end{array}$ & \\
\hline Female & $72 \%$ & 20,5 & $1.3 \%$ & $4 \%$ & $2.7 \%$ & 75 \\
\hline Male & $62.7 \%$ & $30.7 \%$ & $2.7 \%$ & $1.3 \%$ & $2.7 \%$ & 75 \\
\hline Total & $67.3 \%$ & $25.3 \%$ & $2 \%$ & $2.7 \%$ & $2.7 \%$ & 150 \\
\hline
\end{tabular}


Table 4

Responses of the healthcare personel according to the training received

\begin{tabular}{|c|c|c|c|c|c|c|}
\hline \multirow[t]{2}{*}{ Item4 } & \multicolumn{6}{|c|}{ Have you received training about patient rights? } \\
\hline & $\begin{array}{l}18-25 \text { years } \\
(\%)\end{array}$ & $\begin{array}{l}26-35 \text { years } \\
(\%)\end{array}$ & $36-45$ years $(\%)$ & $\begin{array}{l}46 \text { years }<\ldots \\
(\%)\end{array}$ & Total (\%) & \\
\hline Yes & 42.2 & 81.1 & 100 & 100 & 61.3 & \\
\hline No & 57.8 & 18.9 & 0 & 0 & 38.7 & \\
\hline $\begin{array}{l}\text { Total no of } \\
\text { respondents }\end{array}$ & 83 & 53 & 9 & 5 & & \\
\hline \multirow[t]{2}{*}{ Item 4} & \multicolumn{6}{|c|}{ Have you received training about patient rights? } \\
\hline & Dentist(\%) & Nurse(\%) & $\begin{array}{l}\text { Healthcare clerical } \\
\text { worker(\%) }\end{array}$ & $\begin{array}{l}\text { Healthcare } \\
\text { technician(\%) }\end{array}$ & & Other(\%) \\
\hline Yes & 54.9 & 100 & 100 & 53.8 & & 80 \\
\hline No & 45.1 & 0 & 0 & 46.2 & & 20 \\
\hline $\begin{array}{l}\text { Total no of } \\
\text { respondents }\end{array}$ & 113 & 10 & 9 & 13 & & 5 \\
\hline \multirow[t]{2}{*}{ Item 5} & \multicolumn{6}{|c|}{ Do you know about the legal responsibilities of dentists? } \\
\hline & $\begin{array}{l}18-25 \text { years } \\
(\%)\end{array}$ & $\begin{array}{l}26-35 \text { years } \\
(\%)\end{array}$ & $36-45$ years $(\%)$ & $\begin{array}{l}46 \text { years }<\ldots \\
(\%)\end{array}$ & Total $(\%)$ & \\
\hline Yes & 43.4 & 69.8 & 77.8 & 100 & 56.7 & \\
\hline No & 56.6 & 30.2 & 22.2 & 0 & 43.3 & \\
\hline $\begin{array}{l}\text { Total no of } \\
\text { respondents }\end{array}$ & 83 & 53 & 9 & 5 & & \\
\hline \multirow[t]{2}{*}{ Item 5} & \multicolumn{6}{|c|}{ Do you know about the legal responsibilities of dentists? } \\
\hline & Dentist(\%) & Nurse(\%) & $\begin{array}{l}\text { Healthcare clerical } \\
\text { worker(\%) }\end{array}$ & $\begin{array}{l}\text { Healthcare } \\
\text { technician(\%) }\end{array}$ & & Other(\%) \\
\hline \multirow[t]{2}{*}{ Yes } & 61.1 & 40 & 55.6 & 46.2 & & 20 \\
\hline & 38.9 & 60 & 44.4 & 53.8 & & 80 \\
\hline $\begin{array}{l}\text { Total no of } \\
\text { respondents }\end{array}$ & 113 & 10 & 9 & 13 & & 5 \\
\hline
\end{tabular}

Patient right straining had been received by $61.3 \%$ of the participants and not received by $38.7 \%$. Of all the respondents, $56.7 \%$ had information about the legal responsibilities of dentists, and $43.3 \%$ did not. The general findings of the responses given by the participants are shown in Table 1, information about job title is given in Table 2, gender variables in Table 3, and educational level in Table 4.

The responses of "I definitelyagree" and "I agree" were evaluated as positive and "I do not agree" and "I defintely do not agree" were evaluated as negative. The response of "I am undecided" was not taken into consideration.

Accordingly, to the question "have you received training about patient rights?", $57.8 \%$ of the respondents in the 1825 years age group gave a negative response, and as age increased so the rate of having received training increased. Of the nurses and clericalstaff, $100 \%$ had received training, and the rate was observed to fall to $54.9 \%$ for dentists. 
To the question, "do you know about the legal responsibilities of dentists?", 56.6\% of the respondents in the 18-25 years age group gave a negative response and as age increased, so the awareness also increased. Of the dentist group, $61.1 \%$ stated a level of knowledge and this rate fell to $40 \%$ for nurses.

To the statement, "The dentist should give information about complications to a patient who is receiving treatment for the first time", respondents supported the view at approximately $98 \%$ and the opposing rate was $1.5 \%$.

The statement, "The dentist can refuse to see a patient for Professional or personal reasons" was supported by approximately $75 \%$ and opposed by $11 \%$. A positive view was given by $87 \%$ of the dentists, $54 \%$ of the dental technicians and $50 \%$ of the nurses. Males responded positively at the rate of $80 \%$ and females at $71 \%$.

The statement, "The dentist can leave a patient without finishing the treatment for Professional or personal reasons" was supported by approximately $31 \%$ and opposed by $55 \%$.

The statement, "The dentist can prescribe drugs for patients with a gren prescription" was supported by approximately $29 \%$ and opposed by $42 \%$.

The statement, "The dentist can perform treatments such as botox and dermal fillers without a specialist certificate" was supported by $40 \%$ of the male respondents and $24 \%$ of thefemale respondents.

The statement, "The dentist can write a sicknote for a patient without a specialist certificate" was supported by approximately $71 \%$ and opposed by $23 \%$.

The statement, "The dentist can diagnose and prescribe drugs for a disease other than in his ownfield (diabetes, hypertension)" was supported by $9 \%$ and opposed by $81 \%$.

The statement, "Patient consent must be obtained for every kind of medical intervention, and the patient must benefit from these" was supported by $93 \%$ and opposed by $4 \%$. A positive response was given by $100 \%$ of the clericalstaff, dental technicians and other personnel, by $92 \%$ of the dentist sand $80 \%$ of the nurses.

The statement, "The patient has the right to know the identity, position and title of the dentist and other personel who will provide the healthcare and has the right to select and change the personel who will provide the healthcare" was supported by approximately $73 \%$ and opposed by $15 \%$. A positive response was given by $80 \%$ of the auxiliary healthcare personnel, $75 \%$ of the dentists and $70 \%$ of the nurses.

The statement, "There is a responsibility to give full and complete information related to the patient'shealth" was supported almost equally by males (93\%) and females (92\%).

The statement, "Medical interventions can only be made without patient consent because of medical necessity and when precautions must be taken in respect of public health" was supported by approximately $53 \%$ and opposedby $23 \%$. A positive response was given by $67 \%$ of the health care clericalstaff, by $58 \%$ of dentists and $50 \%$ of nurses.

The statement, "The patient can request all kinds of information verb ally and in writing related to their health status" was supported by approximately $90 \%$ and opposed by $5 \%$.

\section{Discussion}

In Turkey, the awareness of patients about their own rights is not at the desired level, for reasons such as lack of information, hesitation by healthcare personnel, and the concern that nothing would change or the situation would become worse fort hem. In a 2007 study conducted in Denizli State Hospital, there sults showed that 76.6\% of patients

Page $11 / 15$ 
had no information about patient rights, $70.3 \%$ would not complain if there were a problem, $81.6 \%$ did not know where they could make a complaint, and $29.4 \%$ thought that even if they made a complaint, nothing would change [20].

However, great progress has been made in recent years with mass communication and education. In a study by Zaybak et al, those living in the city centre were seen to use patient rights at a higher level than those in provincial centres or villages. Information about the subject for illiterate patients was determined to originate from the media [5).

Eksen et al emphasised that females were more sensitive to the subject of patient rights than males [21]. However, another study conducted in Kayseri reported that males had a higher level of knowledge about patient rights than females, and with a greater length of time as a physician, so the level of knowledge increased [22]. Topbaş et al determined that approximately $60 \%$ of physicians in a Medical Faculty knew about patient rights [3]. In a study by Eşiyok et al, it was stated that $43.1 \%$ of dentists had received trainning on the subject of patient rights [23]. In a similar study, Teke et al reported that $55 \%$ of nurses had received training about patient rights [9].

Previous studies have shown that information about patient rights is generally acquired in training after graduation. Ozturk et al concluded that although physicians and nurses working in state hospitals and university hospitals had a high level of knowledge, these rights were not sufficiently respected in university hospitals [24]. When evaluating the approach to patient rights of personnel in first-level healthcare institutions, Ocaktan et al reported that $30.9 \%$ of personnel had acquired knowledge related to patient rights with training before graduation, and $48.8 \%$ had received training after graduation [25].

In the results of the current study, it was seen that approximately $61 \%$ of healthcare personnel had received training on patient rights [ $100 \%$ of those aged 36 years and above, and $42 \%$ of those younger than 25 years. The rate of dentists who had received training was observed to be below average. It was noteworthy that $38.9 \%$ of the dentists did not have knowledge about their own legal responsibilities. When the responses of the healthcare personel to the questionnaire items were examined according to age, there was determined to be a statistically significant difference in the responses to items $4,5,9,11,17$ and $25(p<0.05)$. When the responses were examined according to Professional group, there was determined to be a statistically significant difference in the responses to items 4, 7, 8, 20, 23 and $24(p<0.05)$. In the evaluation of the responses according to gender, the only item showing a statistically significant difference was item 20 $(p<0.05)$.

The results obtained indicated a need for the subjects of patient rights and the legal responsibilites of physicians to be added to the curriculum in further education colleges and faculties training healthcare personnel. There is a significant requirement for in-service training after graduation to be repeated at certain intervals and for scientific activities to be organized. Increasing awareness on the subject of patientrights will contribute to increasing patient satisfaction and improving the quality of service. Patient satisfaction questionnaires applied at certain intervals will be of guidance in taking the necessary precautions.

In the current study, positive responses to the items in the questionnaire were at the rate of approximately $58 \%$ and negative responses were at the level of $33 \%$. The items with a high rate of positive response were item 8 (97.9\%), item 19 $(92.7 \%)$ and item 25 (90\%). These rates provide information that the interventional healthcare services applied in our hospital are patient-centred. The items with a high rate of negative responses were item 18 (81.4\%), item 16 (65.3\%), item 15 (55.4\%) and item 10 (54.6\%). These results demonstrate that the healthcare personel participating in the study act in accordance with ethical rules and the dentists showed sensitivity to remaining with in their legal responsibilities.

The responses to some of the items were extremely interesting: 
The statement, "The dentist can refuse to see a patient for Professional or personal reasons" was supported by $87 \%$ of the dentists, and $50 \%$ of the nurses. Males responded positively at the rate of $80 \%$ and females at $70.7 \%$.

The statement, "Patient consent must be obtained for every kind of medical intervention, and the patient must benefit from these" was supported by $100 \%$ of the clerical staffand dental technicians and although supported by $92 \%$ of the dentists and $80 \%$ of the nurses, these latter two groups were relatively more undecided.

It was extremely interesting that the statement, "The patient has the right to know the identity, position and title of the dentist and other personel who will provide the healthcare and has the right to select and change the personel who will provide the healthcare" was supported by almost three-quarters (73.4\%) of all the personnel.

When the results of this study were compared with previous findings in literature, they were found to be similar. Despite some confusion about the subjects of patient rights and the legal responsibilities of dentists, it was pleasing to see that the levels of awareness were satisfactory.

\section{Conclusion And Recommendations}

The results of this study demonstrated that the healthcare personnel in the Dental Faculty of Dicle University were evaluated relatively positively in respect of their knowledge and attitudes towards patient rights. It was observed that as the level of training increased together with the time spent in the profession, therewas a positive development in the behaviours related to patient rights. Healthcare personel should not forget that the patients are the reason that they are there, and policies should be followed that prioritise patient rights and patient satisfaction.

This study was presented at the 26th International Scientific Congress and Exhibition of Izmir Chamber of Dentists (810 November 2019) in Izmir/Turkey.

\section{Declarations}

\section{Acknowledgements}

We would like to thank all healthcare professionals that agreed to share their perspectives.

\section{Funding}

The authors received no financial support for the research, authorship and/or publication of this article.

\section{Availability of data and materials}

The datasets used and/or analysed during the current study are available from the corresponding author on reasonable request.

\section{Consent for publication}

All participants in this study gave full written informed consent for participation and publication.

\section{Competing interests}

The authors declare that they have no competing interests.

\section{References}


1. Hatemi H. Rights of Special Patient Groups. Journal of Right to Health. 2006; 1: 42-4.

2. Zincir H, Kaya Z. Knowledge levels of the health care employees working at the primary health institutions about patient rights. Journal of Human Sciences. 2009; 6(1): 877-85.

3. Topbas M, Ozlu T, Can G, Bostan S. How much doctors know about patient's rights? Knowledge level of residents and interns in a medical school. Turkiye Klinikleri J Med Ethics. 2005; 13(2): 81-5.

4. Tanriverdi H, Ozmen ME. Effect of patient rights knowledge level on hospital service quality. TSA. 2011; 15(3): 85109.

5. Zaybak A, Eser I, Ismailoglu EG. An analysis of the attitude of patients towards using their patient rights at a university hospital. Florence Nightingale Journal of Nursing. 2012; 20(2): 104-11.

6. Ardahan M. Advocating Role Of Nurses. Journal of Cumhuriyet University School of Nursing. 2003; 7(2): 23-7.

7. Aydın E. Rights of patients in developing countries: the case of Turkey. J MedEthics. 2004; 30: 555-7.

8. Erbil N. Developing scale of patient' rights using attitude. Journal of Human Sciences. 2009; 6(1): 826-37.

9. Teke A, Ucar M, Demir C, Celen O, Karaalp T. Evaluation of knowledge and attitudes of the nurses working in a training hospital about patients' rights, Turkish Armed Forces Preventive Medicine Bulletin 2007; 6(4): 259-66.

10. Woogara J. Patient's right to privacy and dignity in the NHS. Nurs Stand. 2005; 19(18): 33-7.

11. Aslan S, Sezgin M, Hasıloglu SB. The research of the customer satisfaction and factors of constitutive satisfaction in non-governmental health care organizations. Mugla Sıtkı Kocman University Journal of Social and Humanities Sciences Research. 2008; 20: 23-40.

12. Andaleeb SS, Conway C. Customer satisfaction in the restaurant industry: an examination of the transactionspecific model. Journal of services marketing. 2006; 20: 3-11.

13. Ozer A, Cakıl E. Factors affecting patient satisfaction in health care. TAD. 2007; 5(3): 140-143.

14. Hakeri H. Legal responsibilities of dentists, Ankara Bar Association Journal of Health Law Digestas 2009; 1 (1): 19 27.

15. Onsuz MF, Topuzoglu A, Cobek UC, Erturk S, Yılmaz F, Birol S. Satisfaction levels of hospitalized patients in a university hospital in Istanbul, Marmara Medical Journa. 2008; 21/1: 33-4.

16. Margolis S, Almarzouqi S, Revel T, Reed R. Patient satisfaction with primary healthcare service in the United Arab Emirates. International journal for quality healthcare. 2003; 23: 241-9.

17. Mandharia AS, Hassanb AA, Haranb D. Association between perceived health status and satisfaction with quality of care: Evidence from users of primary health care in. Oman Family Practice. 2004; 21 :519-27.

18. Emhan A, Bez Y, Dulek O. The satisfaction levels of the patients admitted to a university hospital. Dicle Medical Journal. 2010; 37(3): 241-7.

19. Bostan S. The investigation of health employees attitudes related patient rights: instances of farabi hospital. Hacettepe Journal of Health Administration. 2007; 10(1): 1-18.

20. Tasci DK. Evaluation of Patients to Received Care after Birth from the Aspect of Patient Rights. Journal of Ataturk University School of Nursing. 2007; 10(3): 26-33.

21. Eksen M, Karadag N, Ishikay C, Karakus A. Determining the level of knowledge of patients about patient rights. International Journal of Human Sciences. 2004; 1(1): 1-12.

22. Ozer A, Gunay O. Knowledge levels of physicians working in kayseri on patient rights. Erciyes Medical Journal 2007; 29(1): 56-63.

23. Esiyok B, Yasar ZF, Turla A. Dentists' knowledge on patient rights, Turkiye Klinikleri J MedSci. 2007; 27: $367-71$.

24. Ozturk H, Yilmaz F, Hindistan S, Cilingir D, Yesilcicek K. Evaluations of doctors, nurses and patıents about patient's rıghts in hospitals. Turkiye Klinikleri J Med Ethics. 2007; 15(3): 145-52. 
25. Ocaktan E, Yildiz A, Ozdemir O. Knowledge and attidudes of health care personel working at abidinpaşa health group directorate. Journal of Ankara University Faculty of Medicine. 2004; 57(3): 129-37.

26. The patient rights guide http://www.tdb.org.tr/ 